\title{
RADICALLY WEAKENING THE LEHMER AND CARMICHAEL CONDITIONS
}

\author{
NATHAN MCNEW
}

\begin{abstract}
Lehmer's totient problem asks if there exist composite integers $n$ satisfying the condition $\varphi(n) \mid(n-1)$, (where $\varphi$ is the Eulerphi function) while Carmichael numbers satisfy the weaker condition $\lambda(n) \mid(n-1)$ (where $\lambda$ is the Carmichael universal exponent function). We weaken the condition further, looking at those composite $n$ where each prime divisor of $\varphi(n)$ also divides $n-1$. (So $\operatorname{rad}(\varphi(n)) \mid(n-1)$.) While these numbers appear to be far more numerous than the Carmichael numbers, we show that their distribution has the same rough upper bound as that of the Carmichael numbers, a bound which is heuristically tight.
\end{abstract}

\section{INTRODUCTION}

Let $\varphi(n)$ denote the Euler totient function of $n$. Lehmer [8] asked whether there exist composite positive integers $n$ such that $\varphi(n) \mid n-1$. Integers which satisfy this "Lehmer Condition" are sometimes referred to as Lehmer numbers, however no examples are known. Cohen and Hagis [3] have shown that any Lehmer numbers would necessarily have at least 14 prime factors, and computations by Pinch 11 show that any examples must be greater than $10^{30}$. Further, Luca and Pomerance [9] have shown that if $\mathcal{L}(x)$ is the number of Lehmer numbers up to $x$ then, as $x \rightarrow \infty$,

$$
\mathcal{L}(x) \leq \frac{x^{1 / 2}}{(\log x)^{1 / 2+o(1)}} .
$$

Carmichael numbers are the composite integers $n$ which satisfy the congruence $a^{n} \equiv a(\bmod n)$ for every integer $a$. (Fermat's little theorem guarantees that any prime number $n$ satisfies this congruence.) Carmichael numbers were first characterized by Korselt [7] in 1899:

Korselt's Criterion. A composite number $n$ is a Carmichael number if and only if $n$ is square-free, and for each prime $p$ which divides $n, p-1$ divides $n-1$.

Korselt did not find any Carmichael numbers, however. The smallest, 561, was found by Carmichael in 1910 2. Carmichael also gave a new characterization of these numbers as those composite $n$ which satisfy $\lambda(n) \mid n-1$, where $\lambda(n)$, the Carmichael lambda function, denotes the size of the largest cyclic subgroup of $(\mathbb{Z} / n \mathbb{Z})^{\times}$.

Since $\lambda(n) \mid \varphi(n)$ for every integer $n$, the Carmichael property can be viewed as a weakening of the Lehmer property. Every Lehmer number would also be a Carmichael number. In contrast to the Lehmer numbers, it is known, due to Alford, Granville and Pomerance [1, that there are infinitely 
many Carmichael numbers. Pomerance 13] also proves an upper bound for the number $C(x)$ of Carmichael numbers up to $x$, namely as $x \rightarrow \infty$,

$$
C(x) \leq x^{1-\{1+o(1)\} \log \log \log x / \log \log x},
$$

and presents a heuristic argument that this is the true size of $C(x)$.

Grau and Oller-Marcén [5] present other possible weakenings of the Lehmer property: looking at the sets of those $n$ such that $\varphi(n) \mid(n-1)^{k}$ for a fixed value of $k$ as well as the set of those $n$ for which $\varphi(n) \mid(n-1)^{k}$ for some $k$, that is all of the primes dividing $\varphi(n)$ also divide $n-1$. Note that this last set is a weakening of both the Lehmer and Carmichael properties, since $\lambda(n)$ and $\varphi(n)$ have the same prime divisors. Our results resolve several conjectures that Grau and Oller-Marcén made in their paper.

We focus primarily on this final set. Let $\kappa(n)=\operatorname{rad}(\varphi(n))$ denote the product of the primes which divide the value $\varphi(n)$. (Note that $\kappa(n)=$ $\operatorname{rad}(\varphi(n))=\operatorname{rad}(\lambda(n))$.) Let $\mathbb{K}(x)$ be the set of composite numbers $n \leq x$ which satisfy $\kappa(n) \mid n-1$, and let $K(x)=|\mathbb{K}(x)|$. (Observe that every prime number $p$ trivially satisfies $\kappa(p) \mid p-1$.)

We prove that the upper bound (11) for $C(x)$ also applies for $K(x)$. We also present upper bounds for the number of $n \in \mathbb{K}(x)$ which are the product of a fixed number of primes, as well as several related conjectures and computations.

\section{The Upper Bound}

The condition for $n$ to be a member of $\mathbb{K}(x)$ is substantially weaker than that required for $n$ to be a Carmichael number, and computations (see Section (5) show that $K(x)$ appears to be substantially greater than $C(x)$. It is therefore somewhat surprising to find that these two functions have the same rough upper bound. Our proof of this fact is similar to the one for $C(x)$ in [13.

Theorem 1. Define $L(x)=\exp \left(\log x \frac{\log \log \log x}{\log \log x}\right)$. Then as $x \rightarrow \infty$,

$$
K(x) \leq \frac{x}{L(x)^{1+o(1)}}
$$

Proof. We consider first those integers $n \leq x$ which have a large prime divisor. Specifically, let $P(n)$ denote the largest prime divisor of $n$, and write $n=m p$ where $p=P(n)$. We restrict our attention to those $n$ with $P(n)>L(x)^{2}$, and let $K^{\prime}(x)=\#\left\{n \in \mathbb{K}(x) \mid P(n)>L(x)^{2}\right\}$.

If $n=m p$ is to satisfy $\kappa(n) \mid n-1$, then we must have $m \leq \frac{x}{p}$, and $m$ must be congruent to $1(\bmod \operatorname{rad}(p-1))$. Thus, for any fixed $p$ there are at most $1+\left\lfloor\frac{x}{p \cdot \operatorname{rad}(p-1)}\right\rfloor$ possibilities for $m$. Requiring $n$ to be composite (thus $m \neq 1$ ) leaves us with at most $\frac{x}{p \cdot \operatorname{rad}(p-1)}$ possibilities. 
Thus we see that

$$
\begin{aligned}
K^{\prime}(x) & =\sum_{\substack{n=m p \leq x \\
p>L(x)^{2} \\
\kappa(n) \mid n-1}} 1 \leq \sum_{p>L(x)^{2}} \frac{x}{p \operatorname{rad}(p-1)} \\
& \leq \sum_{p>L(x)^{2}} \frac{x}{(p-1) \operatorname{rad}(p-1)}
\end{aligned}
$$

Now, we observe that for each prime $p$, the denominator in (2) is a squarefull number, and that any squarefull number can be represented uniquely as $d \operatorname{rad}(d)$ for some integer $d$. We can therefore replace this sum with a sum over all squarefull numbers:

$$
\sum_{p>L(x)^{2}} \frac{x}{(p-1) \operatorname{rad}(p-1)} \leq \sum_{\substack{d>L(x)^{2} \\ d \text { squareful }}} \frac{x}{d}
$$

Using partial summation and the fact that

$$
\sum_{\substack{n \leq x \\ n \text { squareful }}} 1=\frac{\zeta(3 / 2)}{\zeta(3)} x^{1 / 2}+O\left(x^{1 / 3}\right),
$$

we see that

$$
K^{\prime}(x) \leq \sum_{\substack{d>L(x)^{2} \\ d \text { squareful }}} \frac{x}{d} \ll \frac{x}{L(x)} .
$$

We may assume that $n>\frac{x}{L(x)}$, so to prove the theorem, it suffices to count those $n$ with $\frac{x}{L(x)}<n \leq x$ and $P(n) \leq L(x)^{2}$. We denote this count by $K^{\prime \prime}(x)$. Observe that every such $n$ has a divisor $d$ satisfying

$$
\frac{x}{L(X)^{3}}<d \leq \frac{x}{L(x)}
$$

Write $n=m d$, so $m \leq \frac{x}{d}$. Now, if $n=m d$ is to satisfy $\kappa(m d) \mid m d-1$, we have $m \equiv 1(\bmod \kappa(d))$, and since $(n, \kappa(n))=1$ and $\kappa(d) \mid \kappa(n)$ we know $(d, \kappa(d))=1$. Thus the Chinese remainder theorem implies that there are at most $1+\left\lfloor\frac{x}{d \kappa(d)}\right\rfloor$ possibilities for $m$. Thus

$$
K^{\prime \prime}(x) \leq \sum^{\prime}\left(1+\frac{x}{d \kappa(d)}\right) \leq \frac{x}{L(x)}+\sum^{\prime}\left\lfloor\frac{x}{d \kappa(d)}\right\rfloor,
$$

where $\sum^{\prime}$ denotes a sum over $d$ satisfying (3). If $d \kappa(d) \leq x$ and $d$ satisfies (3), then $\kappa(d)<L(x)^{3}$, so that

$$
\begin{aligned}
K^{\prime \prime}(x) & \leq \frac{x}{L(x)}+\sum^{\prime}\left\lfloor\frac{x}{d \kappa(d)}\right\rfloor \\
& \leq \frac{x}{L(x)}+x \sum_{c \leq L(x)^{3}} \frac{1}{c} \sum_{\kappa(d)=c}^{\prime} \frac{1}{d} .
\end{aligned}
$$


We treat the inner sum in (44) by partial summation:

$$
\sum_{\kappa(d)=c}^{\prime} \frac{1}{d}=\frac{L(x)}{x} \sum_{\kappa(d)=c}^{\prime} 1+\int_{\frac{x}{L(x)^{3}}}^{\frac{x}{L(x)}} \frac{1}{t^{2}} \sum_{\substack{\kappa(d)=c \\ d<t}}^{\prime} 1 d t .
$$

We are thus interested in obtaining an upper bound for $\mathcal{K}(t, c)$, the number of $d \leq t$ with $\kappa(d)=c$.

Lemma 1. As $t \rightarrow \infty, \mathcal{K}(t, c) \leq \frac{t}{L(t)^{1+o(1)}}$ uniformly for all $c$.

Before proving the lemma, we see that using this upper bound in (5) gives us

$$
\begin{aligned}
\sum_{\kappa(d)=c}^{\prime} \frac{1}{d} & \leq \frac{L(x)}{x} \mathcal{K}\left(\frac{x}{L(x)}, c\right)+\int_{\frac{x}{L(x)^{3}}}^{\frac{x}{L(x)}} \frac{1}{t^{2}} \mathcal{K}(t, c) d t \\
& \leq L\left(\frac{x}{L(x)}\right)^{-1+o(1)}+\int_{\frac{x}{L(x)^{3}}}^{\frac{x}{L(x)}} \frac{1}{t L(t)^{1+o(1)}} d t \\
& =L(x)^{-1+o(1)}
\end{aligned}
$$

as $x \rightarrow \infty$. This can be used in (4) to see that $K^{\prime \prime}(x) \leq \frac{x}{L(x)^{1+o(1)}}$. The theorem then follows immediately from our estimates of $K^{\prime}(x)$ and $K^{\prime \prime}(x)$.

It thus remains to prove Lemma 11. We may assume that $c \leq t$, otherwise $\mathcal{K}(t, c)=0$. Then, for any $r>0$ we can write:

$$
\begin{aligned}
\mathcal{K}(t, c) & =\sum_{\substack{d \leq t \\
\kappa(d)=c}} 1 \leq t^{r} \sum_{\kappa(d)=c} d^{-r} \\
& \leq t^{r} \sum_{p|d \Rightarrow \operatorname{rad}(p-1)| c} d^{-r}=t^{r} \prod_{\operatorname{rad}(p-1) \mid c} \frac{1}{1-p^{-r}} .
\end{aligned}
$$

Assuming $r \geq 1 / 2+\epsilon$ then

$$
\begin{aligned}
\prod_{\operatorname{rad}(p-1) \mid c} \frac{1}{1-p^{-r}} & =\exp \left(\sum_{\operatorname{rad}(p-1) \mid c}-\log \left(1-p^{-r}\right)\right)=\exp \left(\sum_{\operatorname{rad}(p-1) \mid c} \sum_{n=1}^{\infty} \frac{p^{-n r}}{n}\right) \\
& \left.=\exp \left(\sum_{\operatorname{rad}(p-1) \mid c} p^{-r}\right)+O_{\epsilon}(1)\right) .
\end{aligned}
$$

So we have

$$
\begin{aligned}
\mathcal{K}(t, c) & \ll_{\epsilon} t^{r} \exp \left(\sum_{\operatorname{rad}(p-1) \mid c} p^{-r}\right) \leq t^{r} \exp \left(\sum_{\operatorname{rad}(l) \mid c} l^{-r}\right) \\
= & t^{r} \exp \left(\prod_{p \mid c}\left(1-p^{-r}\right)^{-1}\right) \leq t^{r} \exp \exp \left(\sum_{p \mid c} p^{-r}+O_{\epsilon}(1)\right)
\end{aligned}
$$

by applying this trick a second time. Now, $\sum_{p \mid c} p^{-r}$ is maximized when $c$ is the largest primorial up to $t$, in other words $c=p_{1} p_{2} \cdots p_{k}<t$, where $p_{i}$ 
is the $i$ th prime. Further, if $t$ is sufficiently large, then the prime number theorem implies that $p_{k} \leq 2 \log (t)$ and thus

$$
\sum_{p \mid c} p^{-r} \leq \sum_{p<2 \log (t)} p^{-r}
$$

Choose $r=1-(\log \log \log t) /(\log \log t)$. Thus for large $t$, we may choose $\epsilon=1 / 4$. Then we have $t^{r}=\frac{t}{L(t)}$ and

$$
\sum_{p<2 \log (t)} p^{-r}=O(\log \log t / \log \log \log t) .
$$

Thus

$$
\begin{aligned}
\mathcal{K}(t, c) & \leq t^{r} \exp \exp \left(\sum_{p \mid c} p^{-r}+O_{\epsilon}(1)\right) \\
& =\frac{t}{L(t)} \exp \exp (O(\log \log t / \log \log \log t))=\frac{t}{L(t)^{1+o(1)}},
\end{aligned}
$$

as $t \rightarrow \infty$, which completes the proof of the lemma.

\section{Bounds FOR INTEGERS IN $\mathbb{K}(x)$ WITH $d$ PRIME FACTORS}

Since the integers satisfying our condition have a similar behavior to the Carmichael numbers assymptotically, it is natural to wonder if the behavior of those numbers with a fixed number of prime factors behaves similarly as well. Granville and Pomerance [4] conjecture that the number, $C_{d}(x)$, of Carmichael numbers with exactly $d$ prime factors is $x^{1 / d+o(1)}$ when $d \geq 3$, and as $x \rightarrow \infty$. This has not been proven for any $k$. However, Heath-Brown [6] has shown that $C_{3}(x) \ll_{\epsilon} x^{7 / 20+\epsilon}$. Note that there are no Carmichael numbers with 2 prime factors.

Let $K_{d}(x)=\#\{n \in \mathbb{K}(x), \omega(n)=d\}$ count the integers satisfying our condition up to $x$ with exactly $d$ prime factors. Using the same method as the first part of Theorem 1 we can prove

Theorem 2. Uniformly for $d \geq 2$ we have the bound $K_{d}(x) \ll x^{1-\frac{1}{2 d}}$.

Proof. Consider first those $n>x / 2$. Since $n$ has $d$ prime factors, the largest prime factor must then satisfy $P(n)>(x / 2)^{1 / d}$. Applying the same argument used for integers $n$ with a large prime factor in Theorem 1, we find that the total contribution of such integers is at most $O\left(x^{1-\frac{1}{2 d}}\right)$. Hence, $K_{d}(x)-K_{d}(x / 2) \ll x^{1-\frac{1}{2 d}}$.

Now summing dyadically we have

$$
K_{d}(x)=\sum_{i=0}^{\infty} K_{d}\left(2^{-i} x\right)-K_{d}\left(2^{-i-1} x\right) \ll \sum_{i \geq 0}\left(\frac{x}{2^{i}}\right)^{1-\frac{1}{2 d}} \ll x^{1-\frac{1}{2 d}} .
$$

In contrast to the situation for Carmichael numbers, there do exist numbers satisfying our condition with two prime factors, and we can prove a substantially better bound than that of Theorem 2 in this case. As a matter of fact, their behavior appears to be like that conjectured for Carmichael numbers with a given number of prime factors. 
Theorem 3. The numbers in $\mathbb{K}(x)$ with exactly two prime factors satisfy the bound $K_{2}(x) \leq x^{1 / 2} \exp \left(\frac{2(2 \log x)^{1 / 2}}{\log \log x}\left(1+O\left(\frac{1}{\log \log x}\right)\right)\right)$.

Proof. Write $n=p q \leq x$. Since $\kappa(p q)=\operatorname{rad}((p-1)(q-1))$ and $p q-1=$ $(p-1)(q-1)+(p-1)+(q-1)$ we have that $\kappa(p q) \mid p q-1$ if and only if $\operatorname{rad}(p-1)=\operatorname{rad}(q-1)$. Thus

$$
\begin{aligned}
K_{2}(x) & =\sum_{\substack{p q \leq x \\
\kappa(p q) \backslash p q-1}} 1=\sum_{\substack{p q \leq x \\
\operatorname{rad}(p-1)=\operatorname{rad}(q-1)}} 1 \leq \sum_{\substack{(m+1)(n+1) \leq x \\
\operatorname{rad}(m)=\operatorname{rad}(n)}} 1 \\
\leq & \sum_{\substack{m n \leq x \\
\operatorname{rad}(m)=\operatorname{rad}(n)}} 1 \leq x^{r} \sum_{\substack{m n \leq x \\
\operatorname{rad}(m)=\operatorname{rad}(n)}} \frac{1}{(m n)^{r}}
\end{aligned}
$$

for any $r \geq 0$. We can rewrite this as a double sum:

$$
\begin{aligned}
x_{\substack{m \\
\operatorname{rad}(m)=\operatorname{rad}(n)}} \frac{1}{(m n)^{r}} & =x^{r} \sum_{m \leq x} \frac{1}{m^{r}} \sum_{\substack{n \leq x / m \\
p \mid m \text { iff } p \mid n}} \frac{1}{n^{r}} \leq x^{r} \sum_{m \leq x} \frac{1}{m^{r}} \prod_{p \mid m} \frac{\frac{1}{p^{r}}}{1-\frac{1}{p^{r}}} \\
& =x^{r} \sum_{m \leq x} \frac{1}{m^{r} \operatorname{rad}(m)^{r}} \prod_{p \mid m} \frac{1}{1-p^{-r}} \\
& =x^{r} \sum_{m \leq x} \frac{1}{m^{r} \operatorname{rad}(m)^{r}} \exp \left(\sum_{p \mid m}-\log \left(1-p^{-r}\right)\right) \\
& =x^{r} \sum_{m \leq x} \frac{1}{m^{r} \operatorname{rad}(m)^{r}} \exp \left(\sum_{p \mid m} \sum_{j=1}^{\infty} \frac{p^{-j r}}{j}\right) .
\end{aligned}
$$

As in the proof of Lemma 1, we can replace the condition $p \mid m$ above with $p \leq 2 \log x$, and $m \operatorname{rad}(m)$ by a squareful integer $d$. We also set $r=1 / 2$. Thus:

$$
\begin{aligned}
& x^{1 / 2} \sum_{m \leq x} \frac{1}{m^{1 / 2} \operatorname{rad}(m)^{1 / 2}} \exp \left(\sum_{p \mid m} \sum_{j=1}^{\infty} \frac{p^{-j / 2}}{j}\right) \\
& \quad \leq x^{1 / 2} \exp \left(\sum_{p \leq 2 \log x}\left(p^{-1 / 2}+\frac{1}{2 p}+\sum_{j=3}^{\infty} \frac{p^{-j / 2}}{j}\right)\right) \sum_{\substack{d \leq x^{2} \\
\text { squarefull }}} \frac{1}{d^{1 / 2}} .
\end{aligned}
$$

By the prime number theorem we have

$$
\sum_{p \leq 2 \log x} p^{-1 / 2}=\operatorname{li}\left((2 \log x)^{1 / 2}\right)\left(1+O\left(\frac{1}{\log \log x}\right)\right) .
$$


So we can rewrite the expression above as

$$
\begin{gathered}
x^{1 / 2} \exp \left(\operatorname{li}\left((2 \log x)^{1 / 2}\right)\left(1+O\left(\frac{1}{\log \log x}\right)\right)+\frac{1}{2} \log \log \log x+O(1)\right) \sum_{\substack{d \leq x^{2} \\
d \text { squarefull }}} \frac{1}{d^{1 / 2}} \\
=x^{1 / 2} \exp \left(\frac{2(2 \log x)^{1 / 2}}{\log \log x}\left(1+O\left(\frac{1}{\log \log x}\right)\right)\right) \sum_{\substack{d \leq x^{2} \\
d \text { squarefull }}} \frac{1}{d^{1 / 2}} .
\end{gathered}
$$

By partial summation, we see that

$$
\sum_{\substack{d \leq x^{2} \\ d \text { squarefull }}} \frac{1}{d^{1 / 2}}=O(\log x),
$$

which can be absorbed into the existing error term in our equation, proving the theorem.

Note that if we assume a strong form of the prime $k$-tuples conjecture, due to Hardy and Littlewood, we can show that this is fairly close to the actual size of $K_{2}(x)$. Their conjecture implies that the number of integers $m$ up to $x^{1 / 2}$ with both $m+1$ and $2 m+1$ prime is asymptotically $c x^{1 / 2} /(\log x)^{2}$. Now, whenever both are prime, (and $m \neq 1$ ) we see that $\kappa((m+1)(2 m+1))=\operatorname{rad}\left(2 m^{2}\right)=\operatorname{rad}(m)$, (since $m$ is necessarily even) and $\operatorname{rad}(m) \mid(m+1)(2 m+1)-1$. Thus $K_{2}(x)$ would be at least of order $x^{1 / 2} /(\log x)^{2}$.

\section{4. $k$-LEHMER NUMBERS}

Grau and Oller-Marcén [5] define a $k$-Lehmer number to be an integer $n$ satisfying the condition $\varphi(n) \mid(n-1)^{k}$. (Note that they do not require $n$ to be composite, as we have in our definitions.) In their paper they make several conjectures about the counts of these $k$-Lehmer numbers. Our Theorem 1, which shows in particular that $K(x)=O(\pi(x)$ ) (where $\pi(x)$ is the prime counting function) resolves four of these conjectures, Conjectures 8 (i)-(iv). Namely, this result proves Conjectures 8 (i),(ii) and (iv), while disproving (iii). Our methods, combined with the methods used in [12 to obtain a bound on the Lehmer numbers, can also be used to bound the counts of the $k$-Lehmer numbers.

We let $\mathbb{L}_{k}(x)$ be the set of composite $n$ up to $x$ which satisfy $\varphi(n) \mid(n-1)^{k}$, and $L_{k}(x)=\left|\mathbb{L}_{k}(x)\right|$. (So Grau and Oller-Marcén's function $C_{k}(x)=$ $L_{k}(x)+\pi(x)+1$.)

Theorem 4. For $k \geq 2$ we have $L_{k}(x) \ll_{k} x^{1-\frac{1}{4 k-1}}$.

Proof. We consider three cases, based on the size of the largest prime divisor. We consider first those $n, x^{1-\frac{1}{4 k-1}}<n \leq x$, which have $P(n)<x^{\frac{k}{4 k-1}}$. Any such $n$ will have a divisor $d$ in the range $\left(x^{\frac{k}{4 k-1}}, x^{\frac{2 k}{4 k-1}}\right)$. Write $n=m d$, so $m \leq / d$ and since $\varphi(m d) \mid(m d-1)^{k}$, we see that $(m d-1)^{k} \equiv 0(\bmod \varphi(d))$.

Now, for any positive integer $N$, the number of residue classes $r(\bmod N)$ with $r^{k} \equiv 0(\bmod N)$ is at most $N^{\frac{k-1}{k}}$. Thus, for any fixed $d$, using the fact 
that $(d, \varphi(d))=1$, we see that $m$ must be in one of at most $\varphi(d)^{\frac{k-1}{k}}$ residue classes $\bmod \varphi(d)$, giving us at most

choices for $m$.

$$
\varphi(d)^{\frac{k-1}{k}}\left\lceil\frac{x}{d \varphi(d)}\right] \leq \varphi(d)^{\frac{k-1}{k}}\left(1+\frac{x}{d \varphi(d)}\right)
$$

Summing over all $d$ in the range $I=\left(x^{\frac{k}{4 k-1}}, x^{\frac{2 k}{4 k-1}}\right)$, we get

$$
\begin{aligned}
\sum_{d \in I} \varphi(d)^{\frac{k-1}{k}}\left(1+\frac{x}{d \varphi(d)}\right) & \leq \sum_{d \in I} d^{\frac{k-1}{k}}+\frac{x}{d^{1+\frac{1}{k}}}\left(\frac{d}{\varphi(d)}\right)^{\frac{1}{k}} \\
& \leq \sum_{d \in I} d^{\frac{k-1}{k}}+\sum_{d \in I} \frac{x}{d^{1+\frac{1}{k}}}\left(\frac{d}{\varphi(d)}\right)
\end{aligned}
$$

The first sum is $\ll x^{1-\frac{1}{4 k-1}}$. Now, using partial summation on the second sum and the fact that $\sum_{t \leq x} \frac{t}{\varphi(t)}=O(x)$, we get

$$
\begin{aligned}
\sum_{d \in I} \frac{x}{d^{1+\frac{1}{k}}}\left(\frac{d}{\varphi(d)}\right) & \ll \frac{x}{x^{\left(\frac{2 k}{4 k-1}\right)\left(1+\frac{1}{k}\right)}} \sum_{d \leq x^{\frac{2 k}{4 k-1}}} \frac{d}{\varphi(d)}+x \int_{x^{\frac{k}{4 k-1}}}^{x^{\frac{2 k}{4 k-1}}} \frac{1}{t^{2+\frac{1}{k}}} \sum_{i<t} \frac{t}{\varphi(t)} d t \\
& \ll \frac{x}{x^{\left(\frac{2 k}{4 k-1}\right)\left(1+\frac{1}{k}\right)}}\left(x^{\frac{2 k}{4 k-1}}\right)+x \int_{x^{\frac{k}{4 k-1}}}^{x^{\frac{2 k}{4 k-1}}} \frac{1}{t^{1+\frac{1}{k}}} d t \\
& \ll k x^{1-\frac{2}{4 k-1}}+\frac{x}{x^{\left(\frac{k}{4 k-1}\right)\left(\frac{1}{k}\right)}} \ll x^{1-\frac{1}{4 k-1}} .
\end{aligned}
$$

In the second case we consider those $n$ with $x^{\frac{k}{4 k-1}}<P(n) \leq x^{\frac{2 k}{4 k-1}}$. In this case $n$ again has a divisor in the range $\left(x^{\frac{k}{4 k-1}}, x^{\frac{2 k}{4 k-1}}\right)$, namely $p$, and the above argument applies verbatim.

Finally we've reduced to the case that $P(n)>x^{\frac{2 k}{4 k-1}}$, and the argument used for large primes in our main theorem gives us that the number of $n$ with $\kappa(n) \mid n-1$ and $P(n)>x^{\frac{2 k}{4 k-1}}$ is at most $x^{1-\frac{k}{4 k-1}}$, hence for those $n$ in $\mathbb{L}_{k}(x)$ as well, and our result follows.

We note that it may be possible to improve upon this bound by using techniques developed in more recent papers to obtain better bounds on the Lehmer numbers.

\section{Computations and Conjectures}

Table 1 shows the values of $K(x)$ we computed for increasing powers of 10, compared with values of $C(x)$, computed by Richard Pinch [10. Our computations were done using trial divison, in which a candidate number, $n$, was rejected as soon as soon as it was found to be nonsquarefree, or to have a prime divisor $p$, which failed to satisfy $\operatorname{rad}(p-1) \mid n-1$.

Despite the similar asymptotic bounds that we have for $C(x)$ and $K(x)$, it is clear that $K(x)$ is growing substantially faster, which leads to the conjecture: 
TABLE 1. Values of $C(x)$ and $K(x)$ to $10^{11}$.

\begin{tabular}{|l|l|l|}
\hline$n$ & $C\left(10^{n}\right)$ & $K\left(10^{n}\right)$ \\
\hline 2 & 0 & 4 \\
3 & 1 & 19 \\
4 & 7 & 103 \\
5 & 16 & 422 \\
6 & 43 & 1559 \\
7 & 105 & 5645 \\
8 & 255 & 19329 \\
9 & 646 & 64040 \\
10 & 1547 & 205355 \\
11 & 3605 & 631949 \\
\hline
\end{tabular}

Conjecture 1. $\lim _{x \rightarrow \infty} K(x) / C(x)=\infty$.

At the moment, however, we are unable to prove even the much weaker conjecture:

Conjecture 2. $\lim _{x \rightarrow \infty} K(x)-C(x)=\infty$.

\section{ACKNOWLEDGMENTS}

I would like to thank my advisor, Carl Pomerance, for suggesting the problem and for his invaluable guidance and encouragement throughout the development of this paper.

\section{REFERENCES}

1. W. R. Alford, A. Granville, and C. Pomerance, There are infinitely many Carmichael numbers, Ann. of Math. (2) 139 (1994), no. 3, 703-722.

2. R. D. Carmichael, Note on a new number theory function, Bull. Amer. Math. Soc. 16 (1910), no. 5, 232-238.

3. G. L. Cohen and P. Hagis, Jr., On the number of prime factors of $n$ if $\varphi(n) \mid(n-1)$, Nieuw Arch. Wisk. (3) 28 (1980), no. 2, 177-185.

4. Andrew Granville and Carl Pomerance, Two contradictory conjectures concerning Carmichael numbers, Math. Comp. 71 (2002), no. 238, 883-908.

5. J. M. Grau and A. M. Oller-Marcén, On k-Lehmer numbers, Integers 12 (2012), no. A37.

6. D. R. Heath-Brown, Carmichael numbers with three prime factors, Hardy-Ramanujan J. 30 (2007), 6-12.

7. A. Korselt, Problème chinois, L'intermdiaire math 6 (1899), 143-143.

8. D. H. Lehmer, On Euler's totient function, Bull. Amer. Math. Soc. 38 (1932), no. 10, 745-751.

9. F. Luca, On composite integers $n$ for which $\varphi(n) \mid n-1$, Boletin de la Sociedad Matemática Mexicana 17 (2011), 13-21.

10. R. G. E. Pinch, The Carmichael numbers up to $10^{15}$, Math. Comp. 61 (1993), no. 203, 381-391.

11. _ A note on Lehmers totient problem, Poster presented in ANTS VII, http://www.math.tu-berlin.de/kant/ants/Poster/PinchPoster3.pdf, 2006.

12. C. Pomerance, On composite $n$ for which $\varphi(n) \mid n-1$, Acta Arith. 28 (1975/76), no. 4, $387-389$.

13. plications (Banff, AB, 1988), NATO Adv. Sci. Inst. Ser. C Math. Phys. Sci., vol. 265, Kluwer Acad. Publ., Dordrecht, 1989, pp. 135-161. 
Department of Mathematics, Dartmouth College, Hanover, NH 03755 USA

E-mail address: nathan.g.mcnew@dartmouth.edu 